\title{
ON THE SUPPORT OF A BIMEASURE AND ORTHOGONALLY SCATTERED VECTOR MEASURES
}

\author{
HANNU NIEMI
}

1. Introduction. Let $S$ be a locally compact Hausdorff space. By $\mathscr{K}_{C}(S)$ we denote the linear space of all continuous complex-valued functions defined on $S$ with compact support ${ }^{1}$. Let $K$ be a subset of $S$. By $\mathscr{K}_{C}(S ; K)$ we denote the linear space of all functions $f \in \mathscr{K}_{C}(S)$ such that the support supp $f$ of $f$ is contained in $K$. The topology of $\mathscr{K}_{C}(S ; K)$ is defined by the supremum norm and the topology of $\mathscr{K}_{C}(S)$ is the locally convex inductive limit topology of the Banach spaces $\mathscr{K}_{C}(S ; L)$ relative to the canonical injections $j_{L}: \mathscr{K}_{C}(S ; L) \rightarrow \mathscr{K}_{C}(S), L \subset S, L$ compact. The set of all Radon measures on $S$, i.e., the dual of $\mathscr{K}_{C}(S)$ is denoted by $\mathscr{M}_{C}(S)$.

For two locally compact Hausdorff spaces $S$ and $T$ a bimeasure $B$ on $S \times T$ is defined as a continuous bilinear form $B: \mathscr{K}_{C}(S) \times \mathscr{K}_{C}(T) \rightarrow C$. The set of all bimeasures on $S \times T$ is denoted by $\mathscr{B}_{C}(S \times T)$.

We note that bimeasures are especially studied in the papers of Morse and Transue [5] and Thomas [9]. In our earlier paper [7] we have used Thomas's results on the integration of bimeasures in studying stochastic measures and their so-called covariance bimeasures.

In this paper we shall give a definition of the support of a bimeasure and show that the support of a bimeasure has some very similar properties as the support of a Radon measure.

To justify our definition of the support of a bimeasure we shall consider bimeasures defined by a Radon measure. By a bimeasure defined by a Radon measure we mean a bimeasure $B: \mathscr{K}_{C}(S) \times \mathscr{K}_{C}(T) \rightarrow C$ for which there exists a (unique) Radon measure $\mu: \mathscr{K}_{C}(S \times T) \rightarrow C$ such that

$$
B(f, g)=\mu(f \otimes g), \quad f \in \mathscr{K}_{C}(S), g \in \mathscr{K}_{C}(T) .
$$

We shall show that in this case the support of the bimeasure $B$ coincides with the support of the Radon measure $\mu$.

\footnotetext{
${ }^{1}$ In this paper $R$ stands for the set of all real numbers and $C$ for the set of all complex numbers.
} 
In Section 4 we shall consider bimeasures $B: \mathscr{K}_{C}(S) \times \mathscr{K}_{C}(S) \rightarrow C$ with the property that the support $\operatorname{supp} B$ of $B$ is contained in the set $\Delta_{S \times S}=$ $\{(s, s) \in S \times S \mid s \in S\}$. We shall give two characterizations of such bimeasures. Moreover, we shall show that for such a bimeasure $B$ on $S \times S$ there exists a unique Radon measure $\mu_{B}$ on $S$ such that

$$
B(f, g)=\mu_{B}(f g), \quad f, g \in \mathscr{K}_{C}(S),
$$

and

$$
B(u, v)=\int u v d \mu_{B}
$$

for all $B$-integrable pairs $(u, v)$ of functions $u: S \rightarrow C, v: S \rightarrow C$.

In Section 5 we shall consider orthogonally scattered vector measures. These vector measures are especially studied by Masani [4]. (For further development of the theory of orthogonally scattered measures see e.g. Sundaresan and Woyczynski [8] and Musiał [6]).

We remark that in this paper we consider vector measures as vector valued Radon measures whereas Masani considered them as completely additive vector valued set functions. As an application of our results on the support of a bimeasure we shall show that the basic results proved by Masani can be proved for orthogonally scattered vector valued Radon measures using the bimeasure theory. The basic idea in our proof is the use of bimeasures $B: \mathscr{K}_{C}(S) \times \mathscr{K}_{C}(S) \rightarrow C$ with the property $\operatorname{supp} B \subset \Delta_{S \times S}$.

In this paper we use Thomas's [9] results concerning the integration of bimeasures and vector measures. Thomas has proved these results in the case of real scalars, but the results are valid even in the case of complex scalars. The basic results concerning the integration of bimeasures and vector measures are also presented in our earlier paper [7]. For convenience we here give references to both of these papers.

Note added in proof: We have recently learned that Varopoulos [10, p. 81] has given a definition of the support of a bimeasure.

2. The support of a bimeasure. We begin with the definition of the support of a bimeasure. Before stating the definition we introduce some notation.

Let $U$ (resp. $V$ ) be an arbitrary set and let $E$ (resp. $F$ ) be a linear space of functions $f: U \rightarrow C$ (resp. $g: V \rightarrow C$ ). As usual, the tensor product $E \otimes F$ is considered as the linear space spanned by the functions $f \otimes g: U \times V \rightarrow C,(f \otimes g)(u, v)=f(u) g(v), u \in U, v \in V, f \in E, g \in F$. Moreover, let $A \subset E$ and $B \subset F$. Then $A \otimes B$ stands for the set 
$\{f \otimes g \mid f \in A, g \in B\}$. If $B: E \times F \rightarrow C$ is a bilinear form, then we denote by $\tilde{B}$ the unique linear form defined on $E \otimes F$ satisfying

$$
\tilde{B}(f \otimes g)=B(f, g), \quad f \in E, \quad g \in F .
$$

Throughout the paper $S$ (resp. $T$ ) stands for a locally compact Hausdorff space. If $G_{1} \subset S, G_{2} \subset T$ are open sets, then $\mathscr{K}_{C}\left(G_{1}\right) \otimes \mathscr{K}_{C}\left(G_{2}\right)$ can be considered as a linear subspace of $\mathscr{K}_{C}(S) \otimes \mathscr{K}_{C}(T)$. Suppose that $B$ is a bimeasure on $S \times T$. Then we denote by $\left.\tilde{B}\right|_{G_{1} \times G_{2}}$ the restriction of $\tilde{B}$ to $\mathscr{K}_{C}\left(G_{1}\right) \otimes \mathscr{K}_{C}\left(G_{2}\right)$.

Definition 1. Let $B: \mathscr{K}_{C}(S) \times \mathscr{K}_{C}(T) \rightarrow C$ be a bimeasure. The support of $B, \operatorname{supp} B$, is the complement of the union of the sets $G_{1} \times G_{2}$ such that $\left.\tilde{B}\right|_{G_{1} \times G_{2}}=0$, where $G_{1} \subset S, G_{2} \subset T$ are open.

We recall that the support of a Radon measure $\mu: \mathscr{K}_{C}(S \times T) \rightarrow C$ is defined as the union of the complement of the open sets $G \subset S \times T$ such that $\left.\mu\right|_{G}=0$, where $\left.\mu\right|_{G}$ is the restriction of $\mu$ to the space $\mathscr{K}_{C}(G)$.

We remark that the difference between the definitions of the support of a Radon measure and of a bimeasure is the use of open rectangles instead of arbitrary open sets in the latter definition.

The support of a Radon measure $\mu: \mathscr{K}_{C}(S \times T) \rightarrow C$ can be also characterized as the complement of the largest open subset $G$ of $S \times T$ such that $\left.\mu\right|_{G}=0 \quad\left(\right.$ Bourbaki $\left.[1, \text { p. } 66]^{2}\right)$. We shall show that for every bimeasure $B$ on $S \times T$ the complement of the set $\operatorname{supp} B$ is the largest open subset $G$ of $S \times T$ with the property $\left.\tilde{B}\right|_{G_{1} \times G_{2}}=0$, if $G_{1} \subset S$ and $G_{2} \subset T$ are open sets such that $G_{1} \times G_{2} \subset G$.

First we prove some preliminary results.

$\mathrm{L}$ e m m a 2. Let $B: \mathscr{K}_{C}(S) \times \mathscr{K}_{C}(T) \rightarrow C$ be a bimeasure on $S \times T$ and let $K_{1} \subset S, K_{2} \subset T$ be compact sets such that $K_{1} \times K_{2} \subset S \times T \backslash \operatorname{supp} B$. Then there exist open sets $G_{1}^{j} \subset S, j=1, \ldots, m, G_{2}^{k} \subset T, k=1, \ldots, n$, such that

and

$$
\begin{gathered}
G_{1}^{j} \times G_{2}^{k} \subset S \times T \backslash \operatorname{supp} B,\left.\quad \tilde{B}\right|_{G_{1}^{j} \times G_{2}^{k}}=0, \\
j=1, \ldots, m, k=1, \ldots, n .
\end{gathered}
$$

There exist continuous functions $f_{j}: S \rightarrow[0,1], g_{k}: T \rightarrow[0,1]$ such that $\operatorname{supp} f_{j} \subset G_{1}^{j}, \quad \operatorname{supp} g_{k} \subset G_{2}^{k}, j=1, \ldots, m, k=1, \ldots, n$. Furthermore,

\footnotetext{
${ }^{2}$ Unless otherwise stated we refer to the 2 nd edition of Bourbaki [1].
} 
(3) $\sum_{j=1}^{m} f_{j}(s) \leq 1$ when $s \in S, \sum_{j=1}^{m} f_{j}(s)=1$ when $s \in K_{1}$,

(4) $\sum_{k=1}^{n} g_{k}(t) \leq 1$ when $t \in T, \sum_{k=1}^{n} g_{k}(t)=1$ when $t \in K_{2}$.

Proof. By Definition 1 for every $(s, t) \in K_{1} \times K_{2}$ there exist open sets $G_{1}(s, t), \quad G_{2}(s, t) \quad$ such that $(s, t) \in G_{1}(s, t) \times G_{2}(s, t), \quad G_{1}(s, t) \times G_{2}(s, t)$ $C S \times T \backslash \operatorname{supp} B$ and

$$
\left.\tilde{B}\right|_{G_{1}(s, t) \times G_{2}(s, t)}=0 \text {. }
$$

Since $K_{1} \times K_{2}$ is a compact set, there exists a finite collection $\left(s_{k}, t_{k}\right)$, $k=1, \ldots, M$, of points belonging to $K_{1} \times K_{2}$ such that the sets $G_{1}\left(s_{k}, t_{k}\right) \times G_{2}\left(s_{k}, t_{k}\right), \quad k=1, \ldots, M$, cover $K_{1} \times K_{2}$. Let $s^{\prime} \in K_{1}$. Put

$$
G_{1}\left(s^{\prime}, .\right)=\bigcap_{\left\{k \mid s^{\prime} \in G_{1}\left(s_{k}, t_{k}\right)\right\}} G_{1}\left(s_{k}, t_{k}\right) .
$$

Then $G_{1}\left(s^{\prime},.\right)$ is open and $s^{\prime} \in G_{1}\left(s^{\prime},.\right)$. Moreover,

$$
\left.\tilde{B}\right|_{G_{1}\left(s^{\prime}, .\right) \times G_{2}\left(s_{k}, t_{k}\right)}=0
$$

for all $k$ such that $s^{\prime} \in G_{1}\left(s_{k}, t_{k}\right)$. Let $t^{\prime \prime} \in K_{2}$. Put

$$
G_{2}\left(., t^{\prime \prime}\right)=\underset{\left\{k \mid t^{\prime \prime} \in G_{2}\left(s_{k}, t_{k}\right)\right\}}{\cap} G_{2}\left(s_{k}, t_{k}\right) .
$$

Then $G_{2}\left(., t^{\prime \prime}\right)$ is open and $t^{\prime \prime} \in G_{2}\left(., t^{\prime \prime}\right)$. Moreover,

$$
\left.\tilde{B}\right|_{G_{1}\left(s_{k}, t_{k}\right) \times G_{2}\left(., t^{\prime \prime}\right)}=0
$$

for all $k$ such that $t^{\prime \prime} \in G_{2}\left(s_{k}, t_{k}\right)$. Let $G_{1}^{j}, j=1, \ldots, m,\left(\operatorname{resp} . G_{2}^{k}\right.$, $k=1, \ldots, n)$ be a renumbering of the different sets $G_{1}\left(s^{\prime},.\right), s^{\prime} \in K_{1}$ $\left(\right.$ resp. $\left.G_{2}\left(., t^{\prime \prime}\right), t^{\prime \prime} \in K_{2}\right)$. Then the sets $G_{1}^{j}, j=1, \ldots, m,\left(\operatorname{resp} . G_{2}^{k}\right.$, $k=1, \ldots, n)$ form an open covering of $K_{1}$ (resp. $K_{2}$ ). Moreover, let $j \in\{1, \ldots, m\}$ and $k \in\{1, \ldots, n\}$ be fixed. Then

$$
\left.\tilde{B}\right|_{G_{1}^{j} \times G_{2}^{k}}=0
$$

since for every $(s, t) \in G_{1}^{j} \times G_{2}^{k}$ there exists at least one $k^{\prime} \in\{1, \ldots, M\}$ such that $(s, t) \in G_{1}\left(s_{k^{\prime}}, t_{k^{\prime}}\right) \times G_{2}\left(s_{k^{\prime}}, t_{k^{\prime}}\right)$ and, by construction,

$$
G_{1}^{j} \times G_{2}^{k} \subset G_{1}\left(s_{k^{\prime}}, t_{k^{\prime}}\right) \times G_{2}\left(s_{k^{\prime}}, t_{k^{\prime}}\right) .
$$

Thus the sets $G_{1}^{j}, j=1, \ldots, m, G_{2}^{k}, k=1, \ldots, n$, have the desired properties.

The latter part of the lemma is a direct consequence of a result proved in Bourbaki ([1, pp. 43-44]). 
Th e o r e m 3 . Let $B: \mathscr{K}_{C}(S) \times \mathscr{K}_{C}(T) \rightarrow C$ be a bimeasure. Then $S \times T \backslash \operatorname{supp} B$ is the largest open subset $G$ of $S \times T$ with the property $\left.\tilde{B}\right|_{G_{1} \times G_{2}}=0$ for all open sets $G_{1} \subset S, \quad G_{2} \subset T$ such that $G_{1} \times G_{2} \subset G$.

Proof. Suppose that $G_{1} \subset S, G_{2} \subset T$ are open sets such that $G_{1} \times G_{2} \subset S \times T \backslash \operatorname{supp} B$. We show that $\left.\tilde{B}\right|_{G_{1} \times G_{2}}=0$. Obviously it is enough to show that $\tilde{B}(f \otimes g)=0$, if $f \otimes g \in \mathscr{K}_{C}\left(G_{1}\right) \otimes \mathscr{K}_{C}\left(G_{2}\right), f \in \mathscr{K}_{C}\left(G_{1}\right)$, $g \in \mathscr{K}_{C}\left(G_{2}\right)$. Hence, suppose that $f \in \mathscr{K}_{C}\left(G_{1}\right), g \in \mathscr{K}_{C}\left(G_{2}\right)$ and denote

$$
K_{1}=\operatorname{supp} f, \quad K_{2}=\operatorname{supp} g .
$$

By Lemma 2 there exist open sets $G_{1}^{j} \subset S, G_{2}^{k} \subset T$ and continuous functions $f_{j}: S \rightarrow[0,1], \quad g_{k}: T \rightarrow[0,1], \quad j=1, \ldots, m, \quad k=1, \ldots, n$, satisfying the conditions $(1)-(4)$. Since

we get

$$
f \otimes g=\sum_{j=1}^{m} \sum_{k=1}^{n}\left(f_{j} f\right) \otimes\left(g_{k} g\right),
$$

$$
\tilde{B}(f \otimes g)=\sum_{j=1}^{m} \sum_{k=1}^{n} \tilde{B}\left(\left(f_{j} f\right) \otimes\left(g_{k} g\right)\right)=0 .
$$

Thus $\left.\tilde{B}\right|_{G_{1} \times G_{2}}=0$.

Suppose that $G \subset S \times T$ is an open set such that $\left.\tilde{B}\right|_{G_{1} \times G_{2}}=0$ for all open sets $G_{1} \subset S, G_{2} \subset T$ satisfying the condition $G_{1} \times G_{2} \subset G$. We show that $G \subset S \times T \backslash \operatorname{supp} B$. Let $(s, t) \in G$. Since $G$ is open, there exist open sets $G_{1} \subset S, G_{2} \subset T$ such that $(s, t) \in G_{1} \times G_{2} \subset G$. Therefore

$$
\left.\tilde{B}\right|_{G_{1} \times G_{2}}=0 \text {, }
$$

thus $(s, t)$ is a point of the complement of $\operatorname{supp} B$. It follows that $G \subset S \times T \backslash \operatorname{supp} B$, which proves the theorem.

Let $\mu: \mathscr{K}_{C}(S \times T) \rightarrow C$ be a Radon measure. We recall that $\mu(f)=0$, if $f(s, t)=0$ for all $(s, t)$ belonging to the support of $\mu$ (Bourbaki $[1$, p. 68]). In the following lemma we state an analogous result concerning bimeasures.

L e m m a 4. Let $B: \mathscr{K}_{C}(S) \otimes \mathscr{K}_{C}(T) \rightarrow C$ be a bimeasure. If $f \in \mathscr{K}_{C}(S)$, $g \in \mathscr{K}_{C}(T)$ and if $(f \otimes g)(s, t)=0$ for all $(s, t) \in \operatorname{supp} B$, then $\tilde{B}(f \otimes g)=0$.

Proof. Let $\varepsilon>0$. Denote

and

$$
K_{1}^{\prime}=\operatorname{supp} f, \quad K_{2}^{\prime}=\operatorname{supp} g
$$

$$
U=\{s \in S|| f(s) \mid<\varepsilon\}, \quad V=\{t \in T|| g(t) \mid<\varepsilon\} .
$$

Then the sets $S \backslash U \subset K_{1}^{\prime}, T \backslash V \subset K_{2}^{\prime}$ are compact and $(S \backslash U) \times(T \backslash V)$ $\subset S \times T \backslash \operatorname{supp} B$. Thus, by Lemma 2 , there exist open sets $G_{1}^{j} \subset S$, $G_{2}^{k} \subset T, j=1, \ldots, m, k=1, \ldots, n$, such that 


$$
\bigcup_{j=1}^{m} G_{1}^{j} \supset S \backslash U, \quad \bigcup_{k=1}^{n} G_{2}^{k} \supset T \backslash V
$$

and

$$
\left.\tilde{B}\right|_{G_{1}^{j} \times G_{2}^{k}}=0, \quad j=1, \ldots, m, k=1, \ldots, n .
$$

Moreover, there exist continuous functions $f_{j}: S \rightarrow[0,1], g_{k}: T \rightarrow$ $[0,1]$ such that $\operatorname{supp} f_{j} \subset G_{1}^{j}, \operatorname{supp} g_{k} \subset G_{2}^{k}, j=1, \ldots, m, k=1, \ldots, n$, and such that the conditions (3) and (4) are satisfied (write $K_{1}=S \backslash U$ in (3) and in (4) $K_{2}=T \backslash V$ ). It follows that

$$
\begin{gathered}
\left|\sum_{j=1}^{m} f f_{j}\right| \leq|f|, \quad\left|\sum_{k=1}^{n} g g_{k}\right| \leq|g|, \\
\left|f-\sum_{j=1}^{m} f f_{j}\right|<2 \varepsilon,\left|g-\sum_{k=1}^{n} g g_{k}\right|<2 \varepsilon
\end{gathered}
$$

and

$$
\operatorname{supp}\left(f-\sum_{j=1}^{m} f f_{j}\right) \subset K_{1}^{\prime}, \quad \operatorname{supp}\left(g-\sum_{k=1}^{n} g g_{k}\right) \subset K_{2}^{\prime} .
$$

Since the bilinear form $B: \mathscr{K}_{C}(S) \times \mathscr{K}_{C}(T) \rightarrow C$ is continuous, there exists a constant $M_{K_{1}^{\prime}, K_{2}^{\prime}}$ such that

$$
\left|B\left(h_{1}, h_{2}\right)\right| \leq M_{K_{1}^{\prime}, K_{2}^{\prime}} \sup \left|h_{1}\right| \sup \left|h_{2}\right|
$$

for all $h_{1} \in \mathscr{K}_{C}\left(S ; K_{1}^{\prime}\right), \quad h_{2} \in \mathscr{K}_{C}\left(T ; K_{2}^{\prime}\right)$. It follows that

$$
\begin{aligned}
|B(f, g)| & \leq\left|B\left(f, g-\sum_{k=1}^{n} g g_{k}\right)\right|+\left|B\left(f-\sum_{j=1}^{m} f f_{j}, \sum_{k=1}^{n} g g_{k}\right)\right| \\
& +\left|B\left(\sum_{j=1}^{m} f f_{j}, \sum_{k=1}^{n} g g_{k}\right)\right| \\
& \leq 2 M_{K_{1^{\prime}}, K_{2}{ }^{\prime}} \sup |f| \varepsilon+2 M_{K_{1^{\prime}}, K_{2}{ }^{\prime}} \sup |g| \varepsilon .
\end{aligned}
$$

Thus $\tilde{B}(f \otimes g)=B(f, g)=0$. The lemma is proved.

Let $\mu: \mathscr{K}_{C}(S \times T) \rightarrow C$ be a Radon measure. In this paper we denote by $\operatorname{supp} \mu$ the support of $\mu$. We recall that $\mu$ is an element of the $\sigma\left(\mathscr{M}_{C}(S \times T), \mathscr{K}_{C}(S \times T)\right)$-closed subspace spanned by the Dirac measures $\delta_{(s, t)}, \quad(s, t) \in \operatorname{supp} \mu$, i.e.,

$$
\left\{\delta_{(s, t)} \mid(s, t) \in \operatorname{supp} \mu\right\}^{\perp \perp},
$$

where the orthogonal complements are taken in the duality $\left(\mathscr{K}_{C}(S \times T), \mathscr{M}_{C}(S \times T)\right)$ (Bourbaki [1, p. 71]). In the following theorem we state an analogous result concerning bimeasures. 
We recall that the set of all bimeasures on $S \times T$ is denoted by $\mathscr{B}_{C}(S \times T)$. In the following lines we use the notation $\tilde{\mathscr{B}}_{C}(S \times T)=$ $\left\{\tilde{B} \mid B \in \mathscr{B}_{C}(S \times T)\right\}$.

The or e m 5. Let $B: \mathscr{K}_{C}(S) \times \mathscr{K}_{C}(T) \rightarrow C$ be a bimeasure. Then

$$
\tilde{B} \in\left\{f \otimes g \in \mathscr{K}_{C}(S) \otimes \mathscr{K}_{C}(T) \mid(f \otimes g)(s, t)=0,(s, t) \in \operatorname{supp} B\right\}^{\perp},
$$

where the orthogonal complement is taken in the duality

$$
\left(\tilde{\mathscr{B}}_{C}(S \times T), \mathscr{K}_{C}(S) \otimes \mathscr{K}_{C}(T)\right) .
$$

Proof. Let $B$ be a bimeasure on $S \times T$. Then by Lemma 4 we have $\tilde{B}(f \otimes g)=0$ for all $f \otimes g \in \mathscr{K}_{C}(S) \otimes \mathscr{K}_{C}(T)$ such that $(f \otimes g)(s, t)=0$ for all $(s, t) \in \operatorname{supp} B$, which proves the theorem.

Remark. Let $A \subset S \times T$. In this paper we use the notations

$\pi_{1}(A)=\{s \in S \mid$ there exists $t \in T$ such that $(s, t) \in A\}$,

$\pi_{2}(A)=\{t \in T \mid$ there exists $s \in S$ such that $(s, t) \in A\}$.

Theorem 5 can also be formulated as follows. Let $B: \mathscr{K}_{C}(S) \times \mathscr{K}_{C}(T) \rightarrow C$ be a bimeasure. Then

$$
\tilde{B} \in\left\{\left\{\delta_{s} \mid s \in \pi_{1}(\operatorname{supp} B)\right\}^{\perp} \underline{\otimes}\left\{\delta_{t} \mid t \in \pi_{2}(\operatorname{supp} B)\right\}^{\perp}\right\}^{\perp},
$$

where the orthogonal complements (from left to right) are taken in the dualities $\left(\mathscr{K}_{C}(S), \mathscr{M}_{C}(S)\right),\left(\mathscr{K}_{C}(T), \mathscr{M}_{C}(T)\right)$ and $\left(\tilde{\mathscr{B}}_{C}(S \times T), \mathscr{K}_{C}(S) \otimes \mathscr{K}_{C}(T)\right)$.

3. Bimeasures defined by Radon measures. Suppose that $\mu: \mathscr{K}_{C}(S \times T)$ $\rightarrow C$ is a Radon measure. Then the bilinear form $B: \mathscr{K}_{C}(S) \otimes \mathscr{K}_{C}(T) \rightarrow C$,

$$
B(f, g)=\mu(f \otimes g), \quad f \in \mathscr{K}_{C}(S), g \in \mathscr{K}_{C}(T),
$$

is a bimeasure on $S \times T$. On the other hand, if $B: \mathscr{K}_{C}(S) \times \mathscr{K}_{C}(T) \rightarrow C$ is a bimeasure on $S \times T$, then there does not always exist a Radon measure $\mu$ on $S \times T$ such that (5) is satisfied (see e.g. Edwards [3, pp. 93-95]).

Definition 6 . Let $B: \mathscr{K}_{C}(S) \times \mathscr{K}_{C}(T) \rightarrow C$ be a bimeasure. If there exists a Radon measure $\mu: \mathscr{K}_{C}(S \times T) \rightarrow C$ such that the equation (5) is satisfied, then we say that $B$ can be extended to a Radon measure or that $B$ is defined by a Radon measure. If $B: \mathscr{K}_{C}(S) \times \mathscr{K}_{C}(T) \rightarrow C$ is a bimeasure which is defined by a Radon measure and if $\mu$ is a Radon measure on $S \times T$ satisfying the equation (5), then we say that $\mu$ is a Radon extension of $B$.

Remark. Let us say that a bimeasure $B$ on $S \times T$ has the property $(C)$, if the linear form $\tilde{B}: \mathscr{K}_{C}(S) \otimes \mathscr{K}_{C}(T) \rightarrow C$ is continuous, when the space $\mathscr{K}_{C}(S) \otimes \mathscr{K}_{C}(T)$ carries the topology induced by $\mathscr{K}_{C}(S \times T)$. A bi- 
measure is defined by a Radon measure if and only if it has the property $(C)$. Suppose that a bimeasure $B$ on $S \times T$ has the property $(C)$. Since the space $\mathscr{K}_{C}(S) \otimes \mathscr{K}_{C}(T)$ is dense in $\mathscr{K}_{C}(S \times T)$ (Bourbaki [1, p. 83]), the linear form $\tilde{B}: \mathscr{K}_{C}(S) \otimes \mathscr{K}_{C}(T) \rightarrow C$ can be extended by continuity to a continuous linear form defined on $\mathscr{K}_{C}(S \times T)$ or equivalently to a Radon measure on $S \times T$. Conversely, it is clear that every bimeasure defined by a Radon measure has the property $(C)$. Moreover, since $\mathscr{K}_{C}(S) \otimes \mathscr{K}_{C}(T)$ is dense in $\mathscr{K}_{C}(S \times T)$ the Radon extension of a bimeasure defined by a Radon measure is unique.

Th e o r e m 7. Suppose that a bimeasure $B: \mathscr{K}_{C}(S) \times \mathscr{K}_{C}(T) \rightarrow C$ is defined by a Radon measure. If $\mu_{B}$ is the (unique) Radon extension of $B$, then

$$
\operatorname{supp} B=\operatorname{supp} \mu_{B} .
$$

Proof. First we show that $\operatorname{supp} B \subset \operatorname{supp} \mu_{B}$. Let $(s, t) \in S \times T \backslash \operatorname{supp} \mu_{B}$. Since $\operatorname{supp} \mu_{B}$ is a closed set, there exists an open set $G \subset S \times T$ such that $(s, t) \in G \subset S \times T \backslash \operatorname{supp} \mu_{B}$. Moreover, we can find open sets $G_{1} \subset S$, $G_{2} \subset T$ such that $(s, t) \in G_{1} \times G_{2} \subset G$. By the definition of supp $\mu_{B}$ we get

$$
B(f, g)=\mu_{B}(f \otimes g)=0
$$

for all $f \in \mathscr{K}_{C}\left(G_{1}\right), g \in \mathscr{K}_{C}\left(G_{2}\right)$. Therefore

$$
\left.\tilde{B}\right|_{G_{1} \times G_{2}}=0 \text {, }
$$

which implies that $(s, t) \in S \times T \backslash \operatorname{supp} B$.

On the other hand, let $(s, t) \in S \times T \backslash \operatorname{supp} B$. By Definition 1 there exist open sets $G_{1} \subset S, G_{2} \subset T$ such that $(s, t) \in G_{1} \times G_{2}$ and

$$
\left.\tilde{B}\right|_{G_{1} \times G_{2}}=0 \text {. }
$$

Since $S$ and $T$ are locally compact Hausdorff spaces we can choose the sets $G_{1}$ and $G_{2}$ such that their closures $\bar{G}_{1}$ and $\bar{G}_{2}$ are compact. Let $h \in \mathscr{K}_{C}(S \times T)$ be such that $\operatorname{supp} h \subset G_{1} \times G_{2}$ and let $\varepsilon>0$. Since the space $\mathscr{K}_{C}(S) \times \mathscr{K}_{C}(T)$ is dense in $\mathscr{K}_{C}(S \times T)$ (Bourbaki [1, p. 83]), there exists an open relatively compact set $U \supset \operatorname{supp} h$ and a function $h_{\varepsilon} \in \mathscr{K}_{C}(S) \otimes \mathscr{K}_{C}(T)$ such that $\operatorname{supp} h_{\varepsilon} \subset U$ and

$$
\sup \left|h-h_{\varepsilon}\right|<\varepsilon
$$

(Bourbaki [1, 1st edition pp. 55-56]).

Denote $K_{1}=\pi_{1}(\operatorname{supp} h), \quad K_{2}=\pi_{2}(\operatorname{supp} h)$. Then the sets $K_{1}$ and $K_{2}$ are compact and $K_{1} \subset G_{1}, K_{2} \subset G_{2}$. Therefore there exist continuous functions $h_{1}: S \rightarrow[0,1], \quad h_{2}: T \rightarrow[0,1]$ such that $\operatorname{supp} h_{1} \subset G_{1}$, 
supp $h_{2} \subset G_{2}, \quad h_{1}(\mathrm{~s})=1, \quad s \in K_{1}, \quad h_{2}(t)=1, \quad t \in K_{2} \quad$ (Bourbaki [1, pp. $43-44])$. Since $h_{\varepsilon} \in \mathscr{K}_{C}(S) \otimes \mathscr{K}_{C}(T)$, it has a representation

$$
h_{\varepsilon}=\sum_{k=1}^{p} u_{k} \otimes v_{k}
$$

Define $\quad h_{\varepsilon}^{\prime} \in \mathscr{K}_{C}(S) \otimes \mathscr{K}_{C}(T) \quad$ by setting

$$
h_{\varepsilon}^{\prime}=h_{\varepsilon}\left(h_{1} \otimes h_{2}\right)=\sum_{k=1}^{p} h_{1} u_{k} \otimes h_{2} v_{k} .
$$

Then $\operatorname{supp}\left(h_{1} u_{k}\right) \subset G_{1}$ and $\operatorname{supp}\left(h_{2} v_{k}\right) \subset G_{2}$ for all $k=1, \ldots, p$. Thus

$$
\tilde{B}\left(h_{\varepsilon}^{\prime}\right)=\sum_{k=1}^{p} \tilde{B}\left(h_{1} u_{k} \otimes h_{2} v_{k}\right)=0 .
$$

Moreover,

$$
\begin{aligned}
\sup _{(s, t) \in S \times T}\left|h(s, t)-h_{\varepsilon}^{\prime}(s, t)\right| & \leq \sup _{(s, t) \in S \times T}\left|h(s, t)-h_{\varepsilon}(s, t)\right| \\
& +\sup _{(s, t) \in S \times T}\left|h_{\varepsilon}(s, t)-h_{\varepsilon}^{\prime}(s, t)\right| \\
& \leq \varepsilon+\sup _{(s, t) \notin K_{1} \times K_{z}}\left|h_{\varepsilon}(s, t)-h_{\varepsilon}^{\prime}(s, t)\right| \\
& \leq \varepsilon+2 \varepsilon
\end{aligned}
$$

since

$$
\begin{aligned}
& h_{\varepsilon}^{\prime}(s, t)=h_{\varepsilon}(s, t) \quad \text { when }(s, t) \in K_{1} \times K_{2} \text { and } \\
& \left|h_{\varepsilon}^{\prime}(s, t)\right| \leq\left|h_{\varepsilon}(s, t)\right| \quad \text { when }(s, t) \notin K_{1} \times K_{2} .
\end{aligned}
$$

Since the linear form $\mu_{B}: \mathscr{K}_{C}(S \times T) \rightarrow C$ is continuous and since the set $\bar{G}_{1} \times \bar{G}_{2} \subset S \times T$ is compact and $\operatorname{supp} h \subset G_{1} \times G_{2}, \quad \operatorname{supp} h_{\varepsilon}^{\prime} \subset G_{1} \times G_{2}$; there exists a constant $M>0$ such that

$$
\left|\mu_{B}(h)\right| \leq\left|\mu_{B}\left(h-h_{\varepsilon}^{\prime}\right)\right|+\left|\mu_{B}\left(h_{\varepsilon}^{\prime}\right)\right| \leq 3 M \varepsilon+\left|\tilde{B}\left(h_{\varepsilon}^{\prime}\right)\right|=3 M \varepsilon .
$$

Since the constant $M$ does not depend on the choice of $\varepsilon$, we get $\mu_{B}(h)=0$. Thus $(s, t) \in S \times T \backslash \operatorname{supp} \mu_{B}$, which proves the theorem.

4. Bimeasures concentrated on the diagonal. Let $S$ be a locally compact Hausdorff space. Denote

$$
\Delta_{S \times S}=\{(s, s) \in S \times S \mid s \in S\} .
$$

In this section we consider bimeasures $B: \mathscr{K}_{C}(S) \times \mathscr{K}_{C}(S) \rightarrow C$ satisfying the condition

$$
\operatorname{supp} B \subset \Delta_{S \times S} \text {. }
$$


Our aim is to show that in this case there exists a Radon measure $\mu_{B}$ on $S$ such that

$$
B(f, g)=\int f g d \mu_{B}
$$

for all $B$-integrable pairs $(f, g)$ and that $B$ is defined by a Radon measure.

First we recall how the integral of a bimeasure is defined (for the details see Thomas [9, pp. 144-145] or our paper [7, pp. 20-22]).

We remark that we use the same terminology as Thomas, i.e., we use the terms integral and integrable when Bourbaki uses the terms essential integral and essentially integrable.

If $\mu$ is a Radon measure, then the set of all $\mu$-integrable functions is denoted by $\mathscr{L}_{C}^{1}(\mu)$.

Let $B$ be a bimeasure on $S \times T$. Thus $B$ is a continuous bilinear form defined on $\mathscr{K}_{C}(S) \times \mathscr{K}_{C}(T)$, so that the linear forms $B(., g): \mathscr{K}_{C}(S) \rightarrow C$ and $B(f,):. \mathscr{K}_{C}(T) \rightarrow C$, which are defined by setting

$$
\begin{aligned}
B(., g)(h)=B(h, g), & & h \in \mathscr{K}_{C}(S), \\
B(f, .)(h)=B(f, h), & & h \in \mathscr{K}_{C}(T),
\end{aligned}
$$

are continuous for all $g \in \mathscr{K}_{C}(T), f \in \mathscr{K}_{C}(S)$. Define the linear mappings $\mu_{B}^{r}: \mathscr{K}_{C}(T) \rightarrow \mathscr{M}_{C}(S)$ and $\mu_{B}^{l}: \mathscr{K}_{C}(S) \rightarrow \mathscr{M}_{C}(T)$ by setting

$$
\begin{array}{ll}
\mu_{B}^{r}(g)=B(., g), & g \in \mathscr{K}_{C}(T), \\
\mu_{B}^{l}(f)=B(f, .), & f \in \mathscr{K}_{C}(S) .
\end{array}
$$

If the space $\mathscr{M}_{C}(S)$ (resp. $\left.\mathscr{M}_{C}(T)\right)$ carries the topology $\sigma\left(\mathscr{M}_{C}(S), \mathscr{K}_{C}(S)\right)$ (resp. the topology $\sigma\left(\mathscr{M}_{C}(T), \mathscr{K}_{C}(T)\right)$ ), then the mapping $\mu_{B}^{r}$ (resp. $\mu_{B}^{l}$ ) is a continuous linear mapping, i.e., it is a vector measure with values in $\mathscr{M}_{C}(S)$ (resp. in $\mathscr{M}_{C}(T)$ ). Moreover, the set of the $\mu_{B}^{r}$-integrable functions is

$$
\mathscr{L}_{C}^{1}\left(\mu_{B}^{\prime}\right)=\bigcap_{f \in \mathscr{K}_{C}(S)} \mathscr{L}_{C}^{1}(B(f, .))
$$

If $h \in \mathscr{L}_{C}^{1}\left(\mu_{B}^{r}\right)$, then the integral of $h$ with respect to $\mu_{B}^{r}$ is denoted by $\int h d \mu_{B}^{r}$ or by $B(., h)$ and it satisfies the conditions

$$
\int h d \mu_{B}^{\gamma} \in \mathscr{M}_{C}(S)
$$

and

$$
\left\langle f, \int h d \mu_{B}^{r}\right\rangle=\int h d B(f, .) \quad \text { for all } f \in \mathscr{K}_{C}(S) .
$$

Similarly, the set of the $\mu_{B}^{l}$-integrable functions is 
(8)

$$
\mathscr{L}_{C}^{1}\left(\mu_{B}^{l}\right)=\bigcap_{g \in \mathscr{K}_{C}^{(T)}} \mathscr{L}_{C}^{1}(B(., g)) .
$$

The integral with respect to $\mu_{B}^{l}$ of a function $h \in \mathscr{L}_{C}^{1}\left(\mu_{B}^{l}\right)$ is denoted by $\int h d \mu_{B}^{l}$ or by $B(h,$.$) and it satisfies the conditions$

and

$$
\int h d \mu_{B}^{l} \in \mathscr{M}_{C}(T)
$$

$$
\left\langle g, \int h d \mu_{B}^{l}\right\rangle=\int h d B(., g) \quad \text { for all } g \in \mathscr{K}_{C}(T) .
$$

Definition 8 . Let $B$ be a bimeasure on $S \times T$. If the functions $f: S \rightarrow C$ and $g: T \rightarrow C$ satisfy the conditions

(i) $f \in \mathscr{L}_{C}^{1}\left(\mu_{B}^{l}\right), g \in \mathscr{L}_{C}^{1}\left(\mu_{B}^{r}\right)$

(the Radon measures $B(f,.) \in \mathscr{M}_{C}(T)$ and $B(., g) \in \mathscr{M}_{C}(S)$ are thus defined),

$$
\begin{aligned}
& \text { (ii) } f \in \mathscr{L}_{C}^{1}(B(., g)) \text { and } g \in \mathscr{L}_{C}^{1}(B(f, .)) \text {, } \\
& \text { (iii) } \int f d B(., g)=\int g d B(f, .) \text {; }
\end{aligned}
$$

then we say that the pair $(f, g)$ is integrable with respect to the bimeasure $B$ or in short $B$-integrable and denote

$$
B(f, g)=\int g d B(f, .)=\int f d B(., g) .
$$

Now we go on to the case of bimeasures $B: \mathscr{K}_{C}(S) \times \mathscr{K}_{C}(S) \rightarrow C$ satisfying the condition (6). First we prove a lemma concerning arbitrary bimeasures. The lemma is proved by using a result due to Morse and Transue [5]. For that reason we first introduce some notation.

In this paper we denote by $\mathscr{J}_{+}(S)$ (resp. by $\mathscr{J}_{+}(T)$ ) the set of all lower semi-continuous nonnegative functions $f: S \rightarrow R^{+} \cup\{\infty\}$ (resp. $\left.g: T \rightarrow R^{+} \cup\{\infty\}\right)$, where $R^{+}=\{x \in R \mid x \geq 0\}$.

Let $B: \mathscr{K}_{C}(S) \times \mathscr{K}_{C}(T) \rightarrow C$ be a bimeasure. Define

$$
\begin{aligned}
& B^{*}(h, k)=\sup _{|f| \leq h,|g|<k}|B(f, g)| \text { when } h \in \mathscr{J}_{+}(S), k \in \mathscr{J}_{+}(T), \\
& t \in \mathscr{K}_{C}^{|f| \leq h)} g \in \mathscr{K}_{C}^{(T)} \\
& B^{*}(h, k)=\inf _{\substack{f \geq h, g \geq k \\
f \in \mathscr{J}_{+}(S) g \in \mathscr{J}_{+}(T)}} B^{*}(f, g) \quad \text { when } h: S \rightarrow R^{+}, \quad k: T \rightarrow R^{+}
\end{aligned}
$$

Let $B$ be a bimeasure on $S \times T$. We recall that Theorem 7.1 of Morse and Transue ([5, p. 494]) states that a pair $(f, g)$ of functions $f: S \rightarrow C$, 
$g: T \rightarrow C$ is $B$-integrable if $f \in \mathscr{L}_{C}^{1}\left(\mu_{B}^{l}\right), g \in \mathscr{L}_{C}^{1}\left(\mu_{B}^{r}\right)$ and if $B^{*}(|f|,|g|)<\infty$ (see also Thomas [9, pp. 146-147]).

Before stating the lemma we note that the characteristic function of a set $A$ is denoted by $\chi_{A}$.

L e m ma 9 . Let $B: \mathscr{K}_{C}(S) \times \mathscr{K}_{C}(T) \rightarrow C$ be a bimeasure. If $U \subset S$ and $V \subset T$ are relatively compact Borel sets, then the pair $\left(\chi_{U}, \chi_{V}\right)$ is $B$-integrable.

Proof. First we note that by the relations (7) and (8) it follows that $\chi_{U} \in \mathscr{L}_{C}^{1}\left(\mu_{B}^{l}\right)$ and $\chi_{V} \in \mathscr{L}_{C}^{1}\left(\mu_{B}^{r}\right)$. Furthermore, let $G_{1} \subset S$ and $G_{2} \subset T$ be open relatively compact sets such that $U \subset G_{1}, V \subset G_{2}$. Since $B: \mathscr{K}_{C}(S) \times \mathscr{K}_{C}(T) \rightarrow C$ is a continuous bilinear form, there exists a constant $M>0$ such that

$$
|B(f, g)| \leq M \sup |f| \sup |g|
$$

for all $f \in \mathscr{K}_{C}\left(S ; \bar{G}_{1}\right), g \in \mathscr{K}_{C}\left(T ; \bar{G}_{2}\right)$. Thus

$$
B^{*}\left(\chi_{G_{1}}, \chi_{G_{2}}\right) \leq M .
$$

Moreover, it follows from the definition of $B^{*}\left(\chi_{U}, \chi_{V}\right)$ that

$$
B^{*}\left(\chi_{U}, \chi_{V}\right) \leq B^{*}\left(\chi_{G_{1}}, \chi_{G_{2}}\right) .
$$

The lemma follows then by the result of Morse and Transue mentioned above.

The following theorem is important for us.

Theorem 10. Let $B: \mathscr{K}_{C}(S) \times \mathscr{K}_{C}(S) \rightarrow C$ be a bimeasure on $S \times S$. If

$$
B\left(\chi_{A_{1}}, \chi_{A_{2}}\right)=0
$$

for all relatively compact Borel sets $A_{1}, A_{2} \subset S$ such that $A_{1} \cap A_{2}=\varnothing$, then there exists a unique Radon measure $\mu_{B}: \mathscr{K}_{C}(S) \rightarrow C$ such that

$$
B(f, g)=\mu_{B}(f g)
$$

for all $f, g \in \mathscr{K}_{C}(S)$; where $(f g)(s)=f(s) g(s), \quad s \in S$.

Proof. First we construct the Radon measure $\mu_{B}$. The method is analogous to the standard method used for example in Bourbaki [1, pp. 164-168] or by Thomas [9, pp. 106-107].

Let $K \subset S$ be a compact set and let $U$ be a relatively compact open subset of $S$ such that $K \subset U$. By $\mathscr{E}_{U}$ we denote the linear span of the characteristic functions of the Borel sets $A \subset U$. The topology of $\mathscr{E}_{U}$ is defined by the supremum norm.

For a Borel set $A \subset U$ we define

$$
\tilde{\mu}_{A}=B\left(\chi_{A}, \chi_{A}\right) .
$$


We show that the linear mapping $\mu: \mathscr{E}_{U} \rightarrow C$ defined by setting

$$
\mu(h)=\sum_{k=1}^{n} a_{k} \tilde{\mu}_{A_{k}}, \quad \text { if } h=\sum_{k=1}^{n} a_{k} \chi_{A_{k}} \in \mathscr{E}_{U},
$$

is continuous. First, by assumption the support of the Radon measure $B\left(., \chi_{U}\right)$ is contained in the compact set $\bar{U}$; thus $B\left(., \chi_{U}\right)$ is a bounded Radon measure (Bourbaki [1, p. 70]). Moreover, if

$$
h=\sum_{k=1}^{n} a_{k} \chi_{A_{k}} \in \mathscr{E}_{U}
$$

then, by assumption,

$$
\mu(h)=\sum_{k=1}^{n} a_{k} \int \chi_{A_{k}} d B\left(., \chi_{U}\right) .
$$

Therefore

$$
|\mu(h)| \leq\left\|B\left(., \chi_{U}\right)\right\| \sup \left|\sum_{k=1}^{n} a_{k} \chi_{A_{k}}\right|,
$$

which proves the continuity of $\mu$.

By $\overline{\mathscr{E}}_{U}$ we denote the closure of $\mathscr{E}_{U}$ in the linear space of all bounded functions $f: U \rightarrow C$ carrying the topology defined by the supremum norm. Since the mapping $\mu: \mathscr{E}_{U} \rightarrow C$ is continuous, it can be extended to a uniquely determined continuous linear mapping $\mu: \overline{\mathscr{E}}_{U} \rightarrow C$. Since $\mathscr{K}_{C}(S ; K) \subset \overrightarrow{\mathscr{E}}_{U} \quad($ Bourbaki $[1$, p. 162]) we get, by restriction, a linear mapping $\mu: \mathscr{K}_{C}(S ; K) \rightarrow C$, which is continuous if the space $\mathscr{K}_{C}(S ; K)$ carries the topology defined by the supremum norm.

It follows from the way of defining the mapping $\mu: \mathscr{K}_{C}(S ; K) \rightarrow C$ that its values remain the same if we had chosen, instead of the set $U$, another open relatively compact subset of $S$ containing the compact set $K$.

It follows that the mapping $\mu_{B}: \mathscr{K}_{C}(S) \rightarrow C$,

$$
\mu_{B}(f)=\mu(f), \quad f \in \mathscr{K}_{C}(S),
$$

is a continuous linear mapping, i.e., $\mu_{B}$ is a Radon measure on $S$.

Moreover, it follows from the construction of the Radon measure $\mu_{B}$, that

$$
\int \chi_{A} d \mu_{B}=\tilde{\mu}_{A}
$$

for all relatively compact Borel sets $A \subset S$.

Next we show that

$$
B(f, g)=\mu_{B}(f g) \quad \text { for all } f, g \in \mathscr{K}_{C}(S)
$$


Let $K, L \subset S$ be compact sets and let $U \subset S$ be an open relatively compact set such that $K \cup L \subset U$. It follows from Lemma 9 that all pairs $(u, v) \in \mathscr{E}_{U} \times \mathscr{E}_{U}$ are $B$-integrable. Thus, we can define a bilinear mapping $B^{\prime}: \mathscr{E}_{U} \times \mathscr{E}_{U} \rightarrow C$ by setting

$$
B^{\prime}(u, v)=B(u, v), \quad u, v \in \mathscr{E}_{U} .
$$

We show that $B^{\prime}$ is continuous, if the space $\mathscr{E}_{U}$ carries the topology defined by the supremum norm.

First we note that $B\left(., \chi_{U}\right)$ is a bounded Radon measure, since $\operatorname{supp}\left(B\left(., \chi_{U}\right)\right) \subset \bar{U}$. Moreover, if

$$
u=\sum_{j=1}^{m} a_{1 j} \chi_{A_{1 j}}, \quad v=\sum_{k=1}^{n} a_{2 k} \chi_{A_{2 k}} \in \mathscr{E}_{U},
$$

then, by assumption,

$$
\begin{aligned}
B^{\prime}(u, v) & =\sum_{j=1}^{m} \sum_{k=1}^{n} a_{1 j} a_{2 k} B\left(\chi_{A_{1 j}}, \chi_{A_{2 k}}\right) \\
& =\sum_{j=1}^{m} \sum_{k=1}^{n} a_{1 j} a_{2 k} B\left(\chi_{A_{1 j} \cap A_{2 k}}, \chi_{A_{1 j} \cap A_{2 k}}\right) .
\end{aligned}
$$

It follows that

$$
\begin{aligned}
\left|B^{\prime}(u, v)\right| & =\left|\int \sum_{j=1}^{m} \sum_{k=1}^{n} a_{1 j} a_{2 k} \chi_{A_{1 j} \cap A_{2 k}} d B\left(., \chi_{U}\right)\right| \\
& \leq\left\|B\left(., \chi_{U}\right)\right\| \sup \left|\sum_{j=1}^{m} \sum_{k=1}^{n} a_{1 j} a_{2 k} \chi_{A_{1 j} \cap A_{2 k}}\right| \\
& \leq\left\|B\left(., \chi_{U}\right)\right\| \sup \left|\sum_{j=1}^{m} a_{1 j} \chi_{A_{1 j}}\right| \sup \left|\sum_{k=1}^{n} a_{2 k} \chi_{A_{2 k}}\right|,
\end{aligned}
$$

which proves the continuity of $B^{\prime}$.

The continuity of $B^{\prime}$ implies that it can be extended to a uniquely determined continuous bilinear mapping $B^{\prime}: \overline{\mathscr{E}}_{U} \times \overline{\mathscr{E}}_{U} \rightarrow C$.

We show that the restriction of $B^{\prime}$ to the space $\mathscr{K}_{C}(S ; K) \times \mathscr{K}_{C}(T ; L)$ coincides with $B$.

Suppose $f \in \mathscr{K}_{C}(S ; K)$. Clearly, $B^{*}(|f|,|v|)<\infty$ for all $v \in \mathscr{E}_{U}$. Thus, using again the result of Morse and Transue ([5, p. 494]) mentioned above, we see that the pair $(f, v)$ is $B$-integrable for all $v \in \mathscr{E}_{U}$. Furthermore, since the function $f$ can be uniformly approximated by the functions of $\mathscr{E}_{U}$ (Bourbaki [1, p. 162]) it follows from the definition of the bilinear form $B^{\prime}: \overline{\mathscr{E}}_{U} \times \overline{\mathscr{E}}_{U} \rightarrow C$ using the Radon measure $B(., v)$ that

$$
B^{\prime}(f, v)=\int f d B(., v), \quad v \in \mathscr{E}_{U} .
$$


Furthermore, since the pair $(f, v)$ is $B$-integrable, we get

$$
B^{\prime}(f, v)=\int v d B(f, .), \quad v \in \mathscr{E}_{U} .
$$

As above, we see using the Radon measure $B(f,$.$) that$

thus

$$
B^{\prime}(f, g)=\int g d B(f, .) \text {; }
$$

$$
B^{\prime}(f, g)=B(f, g) .
$$

Suppose $f \in \mathscr{K}_{C}(S ; K), \quad g \in \mathscr{K}_{C}(S ; L)$. We show that

$$
B(f, g)=\mu_{B}(f g) .
$$

Let $\varepsilon>0$. Then there exists $u, v \in \mathscr{E}_{U}$ such that $\sup |u-f|<\varepsilon$, $\sup |v-g|<\varepsilon$ and $\sup |v| \leq 2 \sup |g|$ (Bourbaki [1, p. 162]); thus

$$
|u v-f g| \leq|u v-f v|+|f v-f g|<2 \varepsilon \sup |g|+\varepsilon \sup |f| .
$$

On the other hand, since $u, v \in \mathscr{E}_{U}$, there exist the representations

$$
u=\sum_{j=1}^{m} a_{1 j} \chi_{A_{1 j}}, \quad v=\sum_{k=1}^{n} a_{2 k} \chi_{A_{2 k}} .
$$

Moreover, by the relation (10) we get

$$
\begin{aligned}
B^{\prime}(u, v) & =\sum_{j=1}^{m} \sum_{k=1}^{n} a_{1 j} a_{2 k} B\left(\chi_{A_{1 j}}, \chi_{A_{2 k}}\right) \\
& =\sum_{j=1}^{m} \sum_{k=1}^{n} a_{1 j} a_{2 k} B\left(\chi_{A_{1 j} \cap A_{2 k}}, \chi_{A_{1 j} \cap A_{2 k}}\right) \\
& =\sum_{j=1}^{m} \sum_{k=1}^{n} a_{1 j} a_{2 k} \tilde{\mu}_{A_{1 j} \cap A_{2 k}} \\
& =\int \sum_{j=1}^{m} \sum_{k=1}^{n} a_{1 j} a_{2 k} \chi_{A_{1 j} \cap A_{2 k}} d \mu_{B}=\int h d \mu_{B},
\end{aligned}
$$

where

$$
h=\sum_{j=1}^{m} \sum_{k=1}^{n} a_{1 j} a_{2 k} \chi_{A_{1 j} \cap A_{2 k}} .
$$

Therefore we get by using the relations (9) and (11)

$$
\begin{aligned}
\left|B(f, g)-\mu_{B}(f g)\right| & \leq\left|B(f, g)-B^{\prime}(u, v)\right|+\left|\int h d \mu_{B}-\mu_{B}(f g)\right| \\
& \leq 2 \varepsilon\left\|B\left(., \chi_{U}\right)\right\|(\sup |f|+2 \sup |g|),
\end{aligned}
$$

which proves that $B(f, g)=\mu_{B}(f g)$. 
The uniqueness of the Radon measure $\mu_{B}$ follows immediately from the relation $B(f, g)=\mu_{B}(f g)$ for all $f, g \in \mathscr{K}_{C}(S)$.

The theorem is proved.

Next we show that the conditions stated in Theorem 10 are satisfied for all bimeasures $B$ on $S \times S$ for which $\operatorname{supp} B \subset \Delta_{S \times S}$.

$\mathrm{Lemma}$ 11. Let $B: \mathscr{K}_{C}(S) \times \mathscr{K}_{C}(T) \rightarrow C$ be a bimeasure. If $K \subset S, L \subset T$ are compact sets such that $K \times L \subset S \times T \backslash \operatorname{supp} B$, then

$$
B\left(\chi_{K}, \chi_{L}\right)=0 .
$$

Proof. Note that by Lemma 9 the pair $\left(\chi_{K}, \chi_{L}\right)$ is $B$-integrable. Therefore it is enough to show that

$$
\int \chi_{L} d B\left(\chi_{K}, .\right)=0
$$

Since $K \times L$ is compact and since $K \times L \subset S \times T \backslash \operatorname{supp} B$, there exist by Lemma 2 relatively compact open sets $G_{1}^{j} \subset S, G_{2}^{k} \subset T$ and continuous functions $f_{j}: S \rightarrow[0,1], g_{k}: T \rightarrow[0,1], j=1, \ldots, m, k=1, \ldots, n$, such that the conditions $(1)-(4)$ are satisfied. Denote

$$
\begin{aligned}
G_{1} & =\bigcup_{j=1}^{m} G_{1}^{j}, \quad G_{2}=\bigcup_{k=1}^{n} G_{2}^{k}, \\
h^{\prime} & =\sum_{j=1}^{m} f_{j}, \quad h^{\prime \prime}=\sum_{k=1}^{n} g_{k} .
\end{aligned}
$$

Note that $G_{1} \times G_{2} \subset S \times T \backslash \operatorname{supp} B, \operatorname{supp} h^{\prime} \subset G_{1}$ and $\operatorname{supp} h^{\prime \prime} \subset G_{2}$.

Let $h \in \mathscr{K}_{C}(T)$ be such that $K \times \operatorname{supp} h \subset S \times T \backslash \operatorname{supp} B$. We show that

$$
\int h d B\left(\chi_{K}, .\right)=0 .
$$

Denote $v_{h}=B(., h)$. If $g \in \mathscr{K}_{C}(S)$, then

$$
\left|v_{h}\right|(|g|)=\sup _{\substack{|f| \leq|g| \\ f \in \mathscr{K}_{C}^{(S)}}}\left|v_{h}(f)\right|=\sup _{\substack{|f| \leq|\leq| \\ f \in \mathscr{K}_{C}(S)}}|B(f, h)| .
$$

By Lemma 4 we get $B(f, h)=0$ for all $f \in \mathscr{K}_{C}(S)$ such that $(f \otimes g)(s, t)=0$ for $(s, t) \in \operatorname{supp} B$. Therefore $\left|v_{h}\right|(|g|)=0$ for all $g \in \mathscr{K}_{C}(S)$ satisfying the condition

$$
(g \otimes h)(s, t)=0 \quad \text { for }(s, t) \in \operatorname{supp} B .
$$

Furthermore, since $h^{\prime} \geq \chi_{K} \geq 0, \quad h^{\prime} \in \mathscr{K}_{C}(S)$ and since $\left(h^{\prime} \otimes h\right)(s, t)=0$ for $(s, t) \in \operatorname{supp} B$ we get

$$
\left|v_{h}\right|\left(\chi_{K}\right) \leq\left|v_{h}\right|\left(h^{\prime}\right)=0 .
$$


Therefore

$$
\int \chi_{K} d B(., h)=0
$$

Clearly, the pair $\left(\chi_{K}, h\right)$ satisfies the condition $B^{*}\left(\chi_{K},|h|\right)<\infty$, thus it is $B$-integrable, which implies that

$$
\int h d B\left(\chi_{K}, .\right)=0 .
$$

Denote $v_{K}=B\left(\chi_{K},.\right)$. As above,

$$
\left|v_{K}\right|\left(\chi_{L}\right) \leq\left|v_{K}\right|\left(h^{\prime \prime}\right) .
$$

Since $\quad h^{\prime \prime} \in \mathscr{K}_{C}(T), \quad h^{\prime \prime} \geq 0$ and $K \times \operatorname{supp} h^{\prime \prime} \subset S \times T \backslash \operatorname{supp} B$ we get, using the relation (12),

$$
\left|v_{K}\right|\left(h^{\prime \prime}\right)=\sup _{\substack{|f| \leq h^{\prime \prime} \\ f \in \mathscr{K}_{C}^{(T)}}}\left|\int f d B\left(\chi_{K}, .\right)\right|=0 .
$$

Therefore $\left|v_{K}\right|\left(\chi_{L}\right)=0$, thus

$$
\int \chi_{L} d B\left(\chi_{K}, .\right)=0
$$

which proves the lemma.

L e m m 12. Let $B: \mathscr{K}_{C}(S) \times \mathscr{K}_{C}(T) \rightarrow C$ be a bimeasure. If $U \subset S, V \subset T$ are relatively compact Borel sets such that $U \times V \subset S \times T \backslash$ $\operatorname{supp} B$, then

$$
B\left(\chi_{U}, \chi_{V}\right)=0
$$

Proof. Note that by Lemma 9 the pair $\left(\chi_{U}, \chi_{V}\right)$ is $B$-integrable. Therefore it is enough to show that

$$
\int \chi_{V} d B\left(\chi_{U}, .\right)=0 .
$$

Denote $v_{U}=B\left(\chi_{U},.\right)$. Let $\varepsilon>0$. Since $\chi_{V} \in \mathscr{L}_{C}^{1}\left(v_{U}\right)$ there exists a compact set $L \subset V$ such that

$$
\left|v_{U}\right|\left(\chi_{V \backslash L}\right)<\varepsilon
$$

(Bourbaki [1, p. 152]).

Denote $v_{L}=B\left(., \chi_{L}\right)$. As above, there exists a compact set $K \subset U$ such that

$$
\left|v_{L}\right|\left(\chi_{U \backslash K}\right)<\varepsilon
$$

It follows that 


$$
\left|\int \chi_{V} d B\left(\chi_{U}, .\right)-\int \chi_{L} d B\left(\chi_{U}, .\right)\right|<\varepsilon
$$

and, using the fact that the pair $\left(\chi_{U}, \chi_{L}\right)$ is $B$-integrable,

$$
\begin{aligned}
& \left|\int \chi_{L} d B\left(\chi_{U}, .\right)-\int \chi_{K} d B\left(., \chi_{L}\right)\right|= \\
& \left|\int \chi_{U} d B\left(., \chi_{L}\right)-\int \chi_{K} d B\left(., \chi_{L}\right)\right|<\varepsilon .
\end{aligned}
$$

Moreover, since $K \times L$ is a compact set and since $K \times L \subset S \times T \backslash \operatorname{supp} B$, we get, using Lemma 11 and the considerations made above

$$
\begin{aligned}
\left|\int \chi_{V} d B\left(\chi_{U}, .\right)\right| & \leq\left|\int \chi_{V} d B\left(\chi_{U}, .\right)-\int \chi_{L} d B\left(\chi_{U}, .\right)\right| \\
& +\left|\int \chi_{U} d B\left(., \chi_{L}\right)-\int \chi_{K} d B\left(., \chi_{L}\right)\right| \\
& +\left|\int \chi_{K} d B\left(., \chi_{L}\right)\right| \leq 2 \varepsilon
\end{aligned}
$$

which proves the lemma.

$\mathrm{L} \mathrm{e} \mathrm{m} \mathrm{m} \mathrm{a} \mathrm{13.} \mathrm{Let} B: \mathscr{K}_{C}(S) \times \mathscr{K}_{C}(S) \rightarrow C$ be a bimeasure on $S \times S$. If $\operatorname{supp} B \subset \Delta_{S \times S}$ and if $U, V \subset S$ are relatively compact Borel sets, then

$$
B\left(\chi_{U}, \chi_{V}\right)=B\left(\chi_{U \cap V}, \chi_{U \cap V}\right) .
$$

Proof. Note that by Lemma 9 the pairs $\left(\chi_{U}, \chi_{V}\right)$ and $\left(\chi_{U \cap V}, \chi_{U \cap V}\right)$ are $B$-integrable.

If $U \cap V=\varnothing$, then

$$
U \times V \subset S \times S \backslash \Delta_{S \times S} \subset S \times S \backslash \operatorname{supp} B .
$$

Thus, in this case we get by Lemma 12

$$
B\left(\chi_{U}, \chi_{V}\right)=0
$$

It follows that in the general case we have

$$
\begin{aligned}
B\left(\chi_{U}, \chi_{V}\right) & =B\left(\chi_{U \cap V}, \chi_{V}\right)+B\left(\chi_{U \cap(S \backslash V)}, \chi_{V}\right) \\
& =B\left(\chi_{U \cap V}, \chi_{V}\right)=B\left(\chi_{U \cap V}, \chi_{U \cap V}\right) \\
& +B\left(\chi_{U \cap V}, \chi_{V \cap(S \backslash U)}\right)=B\left(\chi_{U \cap V}, \chi_{U \cap V}\right) .
\end{aligned}
$$

The following theorem is one of the main results in this paper.

The or e m 14. Let $B: \mathscr{K}_{C}(S) \times \mathscr{K}_{C}(S) \rightarrow C$ be a bimeasure on $S \times S$. Then supp $B \subset \Delta_{S \times S}$ if and only if there exists a unique Radon measure $\mu_{B}: \mathscr{K}_{C}(S) \rightarrow C$ such that 


$$
B(f, g)=\mu_{B}(f g) \quad \text { for all } f, g \in \mathscr{K}_{C}(S) .
$$

Proof. It is clear that in case the Radon measure $\mu_{B}$ on $S$ satisfying the relation (13) exists, it is uniquely determined.

Suppose the bimeasure $B$ on $S \times S$ is defined by the relation (13), where $\mu_{B}$ is a given Radon measure on $S$. Then $f g=0$ for all $f, g \in \mathscr{K}_{C}(S)$ such that $\operatorname{supp} f \cap \operatorname{supp} g=\varnothing$. Thus $B(f, g)=0$, which proves that $\operatorname{supp} B \subset \Delta_{S \times S}$.

On the other hand, if $B: \mathscr{K}_{C}(S) \times \mathscr{K}_{C}(S) \rightarrow C$ is such a bimeasure that supp $B \subset \Delta_{S \times S}$. Then it follows from Lemma 13 that the assumptions stated in Theorem 10 are satisfied, which proves the theorem.

The following characterization is a direct consequence of Theorem 10, Theorem 14 and Lemma 13.

The o r e m 15. Let $B: \mathscr{K}_{C}(S) \times \mathscr{K}_{C}(S) \rightarrow C$ be a bimeasure on $S \times S$. Then $\operatorname{supp} B \subset \Delta_{S \times S}$ if and only if

$$
B\left(\chi_{A_{1}}, \chi_{A_{2}}\right)=0
$$

for all relatively compact Borel sets $A_{1}, A_{2} \subset S$ such that $A_{1} \cap A_{2}=\varnothing$.

Next we consider some special properties of the bimeasures $B$ on $S \times S$ for which $\operatorname{supp} B \subset \Delta_{S \times S}$.

Th e or e m 16. Let $B: \mathscr{K}_{C}(S) \times \mathscr{K}_{C}(S) \rightarrow C$ be a bimeasure on $S \times S$. If supp $B \subset \Delta_{S \times S}$, then $B$ is defined by a Radon measure.

Proof. It is enough to show that the linear form $\tilde{B}: \mathscr{K}_{C}(S) \otimes \mathscr{K}_{C}(S) \rightarrow C$ is continuous, when $\mathscr{K}_{C}(S) \otimes \mathscr{K}_{C}(S)$ carries the topology induced by $\mathscr{K}_{C}(S \times S)$.

Since $\operatorname{supp} B \subset \Delta_{S \times S}$, there exists by Theorem 14 a unique Radon measure $\mu_{B}$ on $S$ such that

$$
B(f, g)=\mu_{B}(f g) \quad \text { for all } f, g \in \mathscr{K}_{C}(S) .
$$

Suppose

$$
h=\sum_{k=1}^{n} f_{k} \otimes g_{k} \in \mathscr{K}_{C}(S) \otimes \mathscr{K}_{C}(S)
$$

Then

$$
\tilde{B}(h)=\sum_{k=1}^{n} B\left(f_{k}, g_{k}\right)=\mu_{B}\left(\sum_{k=1}^{n} f_{k} g_{k}\right)
$$

Moreover, since

$$
\sum_{k=1}^{n} f_{k} g_{k} \in \mathscr{K}_{C}(S)
$$

and since $\mu_{B}$ is a Radon measure on $S$, there exists a constant $M$ such that 


$$
|\tilde{B}(h)|=\left|\mu_{B}\left(\sum_{k=1}^{n} f_{k} g_{k}\right)\right| \leq M \sup \left|\sum_{k=1}^{n} f_{k} g_{k}\right| .
$$

It follows that

$$
\begin{aligned}
|\tilde{B}(h)| & =\left|\tilde{B}\left(\sum_{k=1}^{n} f_{k} \otimes g_{k}\right)\right| \leq M \sup _{s \in S}\left|\sum_{k=1}^{n} f_{k}(s) g_{k}(s)\right| \\
& \leq M \sup _{\left(s, s^{\prime}\right) \in S \times S}\left|\sum_{k=1}^{n}\left(f_{k} \otimes g_{k}\right)\left(s, s^{\prime}\right)\right|
\end{aligned}
$$

which proves the theorem.

Let $B: \mathscr{K}_{C}(S) \times \mathscr{K}_{C}(S) \rightarrow C$ be a bimeasure. The following theorem gives a characterization of $B$-integrable functions in case $\operatorname{supp} B \subset \Delta_{S \times S}$. For the theorem we recall some definitions.

Let $\mu$ be a Radon measure on $S$. A function $f: S \rightarrow C$ is called locally $\mu$-integrable if $f g \in \mathscr{L}_{C}^{1}(\mu)$ for all $g \in \mathscr{K}_{C}(S)$. We remark that for a locally $\mu$-integrable function $f: S \rightarrow C$ the mapping $f . \mu: \mathscr{K}_{C}(S) \rightarrow C$,

$$
(f \cdot \mu)(g)=\int f g d \mu, \quad g \in \mathscr{K}_{C}(S),
$$

is a Radon measure, which is called the measure with density $f$ with respect to $\mu$ (for the details see Bourbaki [2, pp. 41-44]).

In the proof of the following theorem we use a result proved in Bourbaki [2, p. 47]. It states: For a Radon measure $\mu$ on $S$ and for a locally $\mu$ -integrable function $u: S \rightarrow C$ one has $f \in \mathscr{L}_{C}^{1}(u \cdot \mu)$ if and only if $u f \in \mathscr{L}_{C}^{1}(\mu)$; and in this case

$$
\int f d(u \cdot \mu)=\int f u d \mu .
$$

Th e or e m 17. Let $B: \mathscr{K}_{C}(S) \times \mathscr{K}_{C}(S) \rightarrow C$ be a bimeasure on $S \times S$. Let $\operatorname{supp} B \subset \Delta_{S \times S}$ and let $\mu_{B}$ be the unique Radon measure on $S$ satisfying

$$
B(f, g)=\mu_{B}(f g) \quad \text { for all } f, g \in \mathscr{K}_{C}(S) .
$$

A pair $(u, v)$ of functions $u: S \rightarrow C, v: S \rightarrow C$ is B-integrable if and only if $u$ and $v$ are locally $\mu_{B}$-integrable and $u v \in \mathscr{L}_{C}^{1}\left(\mu_{B}\right)$. In this case

$$
B(u, v)=\int u v d \mu_{B} .
$$

Proof. Since

$$
B(f, g)=\mu_{B}(f g) \quad \text { for all } f, g \in \mathscr{K}_{C}(S),
$$

we get

$$
B(f, .)=f \cdot \mu_{B} \text { and } B(., g)=g \cdot \mu_{B},
$$


when $f, g \in \mathscr{K}_{C}(S)$. Moreover, it follows that a function $w: S \rightarrow C$ is locally $\mu_{B}$-integrable if and only if it is $B(f,$.$) -integrable for all f \in \mathscr{K}_{C}(S)$, or equivalently, if and only if it is $B(., g)$-integrable for all $g \in \mathscr{K}_{C}(S)$. Therefore $w$ is locally $\mu_{B}$-integrable if and only if $w \in \mathscr{L}_{C}^{1}\left(\mu_{B}^{l}\right)$, or equivalently, if and only if $w \in \mathscr{L}_{C}^{1}\left(\mu_{B}^{r}\right)$. Moreover, in this case we get, using the result of Bourbaki [2, p. 47] mentioned above,

$$
\begin{array}{rlrl}
\int w d B(f, .) & =\int w f d \mu_{B}, & & f \in \mathscr{K}_{C}(S), \\
\int w d B(., g) & =\int w g d \mu_{B}, & g \in \mathscr{K}_{C}(S) .
\end{array}
$$

Suppose the pair $(u, v)$ of functions $u: S \rightarrow C, v: S \rightarrow C$ is $B$ -integrable. The considerations above show that $u$ and $v$ are locally $\mu_{B}$ -integrable. Thus, it is enough to show that

$$
\int v d B(u, .)=\int u v d \mu_{B}
$$

Since the Radon measure $B(u,$.$) is defined by the relation$

$$
B(u, .)(g)=\int u d B(., g), \quad g \in \mathscr{K}_{C}(S),
$$

and since $u$ is locally $\mu_{B}$-integrable, it follows that $B(u,)=.u \cdot \mu_{B}$. By assumption $(u, v)$ is $B$-integrable, thus $v \in \mathscr{L}_{C}^{1}(B(u,)$.$) . Again by$ the result of Bourbaki [2, p. 47], we get $u v \in \mathscr{L}_{C}^{1}\left(\mu_{B}\right)$ and

$$
B(u, v)=\int v d B(u, .)=\int u v d \mu_{B} .
$$

On the other hand, suppose the functions $u: S \rightarrow C, v: S \rightarrow C$ are locally $\mu_{B}$-integrable and $u v \in \mathscr{L}_{C}^{1}\left(\mu_{B}\right)$. It follows from the considerations made above that

$$
u \in \mathscr{L}_{C}^{1}\left(\mu_{B}^{l}\right), \quad v \in \mathscr{L}_{C}^{1}\left(\mu_{B}^{r}\right) .
$$

Thus, the theorem is proved if we show that

$$
\int v d B(u, .)=\int u d B(., v) \text {. }
$$

By symmetry it is enough to show that

$$
\int v d B(u, .)=\int u v d \mu_{B} .
$$

Also in this case we have $B(u,)=.u \cdot \mu_{B}$. By assumption $u v \in \mathscr{L}_{C}^{1}\left(\mu_{B}\right)$. Thus, using the result of Bourbaki [2, p. 47] we get $v \in \mathscr{L}_{C}^{1}(B(u,)$.$) and$ 


$$
\int v d B(u, .)=\int u v d \mu_{B}
$$

The theorem is proved.

5. An application to orthogonally scattered vector measures. In this chapter $H$ stands for a Hilbert space with complex scalars. Moreover, by $(. \mid$. ) we denote the inner product and by $\|$. $\|$ the norm of $H$.

Let $\Lambda$ be an arbitrary set and let $\mathscr{P}$ be a semiring (or a pre-ring) of subsets of $\Lambda$. We recall that Masani [4, pp. 63-64] defined an orthogonally scattered vector measure (with values in a Hilbert space) as a completely additive set function $\mu: \mathscr{P} \rightarrow H$ such that

$$
\left(\mu\left(A_{1}\right) \mid \mu\left(A_{2}\right)\right)=0 \quad \text { for all } A_{1}, A_{2} \in \mathscr{P}, A_{1} \cap A_{2}=\varnothing
$$

(see also the definition given by Sundaresan and Woyczynski [8, p. 345]).

In this section we define an orthogonally scattered vector valued Radon measure (with values in a Hilbert space) and give two characterizations of such vector measures. Moreover, we show that Masani's basic results for orthogonally scattered vector measures can be proved for orthogonally scattered vector valued Radon measures by using the results concerning bimeasures $B: \mathscr{K}_{C}(S) \times \mathscr{K}_{C}(S) \rightarrow C$ with the property $\operatorname{supp} B \subset \Delta_{S \times S}$ proved in Section 4.

First we recall some results and definitions concerning vector measures with values in a Hilbert space and their integration. For the details see Thomas [9, pp. 65-82] or our paper [7, pp. 15-17].

A continuous linear mapping $\mu: \mathscr{K}_{C}(S) \rightarrow H$ is called an $H$-valued vector measure on $S$ or a vector measure on $S$ with values in $H$. If $\mu$ is an $H$-valued vector measure on $S$, then we denote by $\mathscr{L}_{C}^{1}(\mu)$ the set of all $\mu$-integrable functions.

$\mathrm{D}$ efin ition 18. Let $\mu$ be an $H$-valued vector measure on $S$. Then the (continuous) bilinear form $B: \mathscr{K}_{C}(S) \times \mathscr{K}_{C}(S) \rightarrow C$

$$
B(f, g)=(\mu(f) \mid \mu(\bar{g})), \quad f, g \in \mathscr{K}_{C}(S),
$$

is called the bimeasure defined by $\mu$.

Before stating the following lemma, which is due to Thomas [9, p. 101], we recall that a function $f: S \rightarrow C$ is said to be a Borel function, if $f^{-1}(G)$ is a Borel set in $S$ for all open sets $G \subset C$.

$\mathrm{L}$ e $\mathrm{m} \mathrm{m}$ a 19. If $\mu$ is an $H$-valued vector measure on $S$, then every Borel function $f: S \rightarrow C$ with compact support is $\mu$-integrable.

The following result is proved in our paper [7, pp. 29-30] by using a different terminology, i.e., we considered there stochastic measures and their covariance bimeasures instead of vector measures with values in 
a Hilbert space and bimeasures defined by these vector measures. The difference is only terminological.

Before stating the lemma we remark that for an $H$-valued vector measure $\mu$ we have

$$
\int f d \mu \in H \quad \text { for all } f \in \mathscr{L}_{C}^{1}(\mu) \text {. }
$$

$\mathrm{L} \mathrm{e} \mathrm{m} \mathrm{ma} \mathrm{20.} \mathrm{Let} \mu$ be an H-valued vector measure on $S$ and let $f: S \rightarrow C, g: S \rightarrow C$ be $\mu$-integrable functions. If $B$ is the bimeasure defined by $\mu$, then the pair $(f, \bar{g})$ is B-integrable and

$$
B(f, \bar{g})=\left(\int f d \mu \mid \int g d \mu\right) .
$$

Now we go on to the case of orthogonally scattered vector measures.

Definition 21. An $H$-valued vector measure $\mu$ on $S$ is called orthogonally scattered, if

$$
(\mu(f) \mid \mu(g))=0
$$

for all $f, g \in \mathscr{K}_{C}(S)$ such that $\operatorname{supp} f \cap \operatorname{supp} g=\varnothing$.

Next we give two characterizations of orthogonally scattered vector mesures.

Th e or e m 22. Let $\mu$ be an H-valued vector measure on $S$ and let $B$ be the bimeasure defined by $\mu$. Then $\mu$ is orthogonally scattered if and only if $\operatorname{supp} B \subset \Delta_{S \times S}$.

Proof. Suppose $\mu$ is an orthogonally scattered vector measure on $S$ with values in $H$. If $s, s^{\prime} \in S, s \neq s^{\prime}$, then there exist relatively compact open sets $G_{1}, G_{2} \subset S$ such that $\left(s, s^{\prime}\right) \in G_{1} \times G_{2} \subset S \times S \backslash \Delta_{S \times S}$. Moreover, since $\mu$ is orthogonally scattered, we get

$$
\left.\tilde{B}\right|_{G_{1} \times G_{2}}=0 \text {. }
$$

Thus, $\left(s, s^{\prime}\right) \in S \times S \backslash \operatorname{supp} B$, which proves that

$$
\operatorname{supp} B \subset \Delta_{S \times S} .
$$

On the other hand, suppose $\mu$ is an $H$-valued vector measure on $S$ such that the bimeasure $B$ defined by $\mu$ satisfies the condition $\operatorname{supp} B \subset \Delta_{S \times S}$. If $f, g \in \mathscr{K}_{C}(S)$ are such that $\operatorname{supp} f \cap \operatorname{supp} g=\varnothing$, then $(f \otimes \bar{g})(s, s)=0$ for all $s \in S$. Thus, by Lemma 4 ,

$$
(\mu(f) \mid \mu(g))=B(f, \bar{g})=0 ;
$$

which proves that $\mu$ is orthogonally scattered.

The theorem is proved. 
Th e o r e m 23. An $H$-valued vector measure $\mu$ on $S$ is orthogonally scattered if and only if

$$
\left(\int \chi_{A_{1}} d \mu \mid \int \chi_{A_{2}} d \mu\right)=0
$$

for all relatively compact Borel sets $A_{1}, A_{2} \subset S$ such that $A_{1} \cap A_{2}=\varnothing$.

Proof. Let $B$ be the bimeasure defined by $\mu$. Suppose $A_{1}, A_{2} \subset S$ are relatively compact Borel sets. By Lemma 19 we have $\chi_{A_{1}}, \chi_{A_{2}} \in \mathscr{L}_{C}^{1}(\mu)$. Moreover, by Lemma 20

$$
\left(\int \chi_{A_{1}} d \mu \mid \int \chi_{A_{2}} d \mu\right)=B\left(\chi_{A_{1}}, \chi_{A_{2}}\right)
$$

Thus, the theorem is a direct consequence of Theorem 22 and Theorem 15.

The following theorem is one of the main results in this paper. It is analogous to Theorem 5.9 of Masani [4].

For the theorem we recall that $\mathscr{L}_{C}^{2}(v)$ is the closure of $\mathscr{K}_{C}(S)$ in the space $^{3}$

$$
\mathscr{F}_{C}^{2}(v)=\left\{f: S \rightarrow C|| v \mid \cdot\left(|f|^{2}\right)<\infty\right\} ;
$$

the topology of $\mathscr{F}_{C}^{2}(v)$ is the locally convex topology defined by the seminorm $N_{2, v}(f)=|v|^{\bullet}\left(|f|^{2}\right)^{1 / 2}, \quad f \in \mathscr{F}_{C}^{2}(v)$. Moreover, the space $\mathscr{L}_{C}^{1}(\mu)$ is the closure of $\mathscr{K}_{C}(S)$ in the space ${ }^{4}$

$$
\mathscr{F}_{C}(\mu)=\left\{f: S \rightarrow C \mid \mu^{\bullet}(|f|)<\infty\right\} ;
$$

the topology of $\mathscr{F}_{C}(\mu)$ is the locally convex topology defined by the seminorm $N_{1, \mu}(f)=\mu^{\bullet}(|f|), f \in \mathscr{F}_{C}(\mu)$.

Th e o r e m 24. Let $\mu$ be an orthogonally scattered $H$-valued vector measure on $S$. Then there exists a unique Radon measure $v$ on $S$ such that

$$
(\mu(f) \mid \mu(g))=v(f \bar{g})
$$

for all $f, g \in \mathscr{K}_{C}(S)$. The Radon measure $v$ is real-valued and positive, i.e., $\overline{v(\bar{f})}=v(f)$ for all $f \in \mathscr{K}_{C}(S)$ and $v(f) \geq 0$ for all $f \in \mathscr{K}_{C}(S)$, $f \geq 0$. Furthermore,

$$
\mu^{\bullet}(f) \leq|v| \cdot\left(f^{2}\right)^{1 / 2} \leq 2^{1 / 2} \mu^{\bullet}(f)
$$

for all $f: S \rightarrow R^{+} \cup\{\infty\}, \quad \mathscr{L}_{C}^{1}(\mu)=\mathscr{L}_{C}^{2}(v)$ and

$$
\left(\int f d \mu \mid \int g d \mu\right)=\int f \bar{g} d v
$$

for all $f, g \in \mathscr{L}_{C}^{1}(\mu)$.

\footnotetext{
3 For the details see Bourbaki [2, pp. 7-9]; note the terminological difference.

${ }^{4}$ For the details see Thomas [9, pp. $\left.65-67\right]$ or our paper [7, pp. 15-16].
} 
Proof. The existence and uniqueness of the Radon measure $v: \mathscr{K}_{C}(S) \rightarrow C$ with the property

$$
(\mu(f) \mid \mu(g))=v(f \bar{g}), \quad f, g \in \mathscr{K}_{C}(S),
$$

follows from Theorem 22 and Theorem 14.

Let $f \in \mathscr{K}_{C}(S)$ and let $U \subset S$ be an open relatively compact set containing supp $f$. Then there exists a continuous function $g: S \rightarrow[0,1]$ such that $\operatorname{supp} g \subset U$ and $g(s)=1$ when $s \in \operatorname{supp} f$. It follows that

$$
(\mu(f) \mid \mu(g))=v(f \bar{g})=v(f) .
$$

On the other hand

$$
\overline{v(\bar{f})}=\overline{v(g \bar{f})}=\overline{(\mu(g) \mid \mu(f))}=(\mu(f) \mid \mu(g)),
$$

which proves that $v$ is real-valued.

Moreover, for all $f \in \mathscr{K}_{C}(S), f \geq 0$; we have

$$
v(f)=v\left(f^{1 / 2} f^{1 / 2}\right)=\left(\mu\left(f^{1 / 2}\right) \mid \mu\left(f^{1 / 2}\right)\right) \geq 0 ;
$$

which proves that $v$ is positive.

Next we show that the relation (14) is valid.

Suppose $f \in \mathscr{J}_{+}(S)$. Then

$$
\begin{aligned}
|v| \cdot\left(f^{2}\right) & =\sup _{\substack{0 \leq h \leq f^{2} \\
h \in \mathscr{K}_{C}(S)}}|v|(h)=\sup _{\substack{0 \leq h \leq f^{2} \\
h \in \mathscr{K}_{C}(S)}} \sup _{\substack{|g| \leq h \\
g \in \mathscr{K}_{C}(S)}}|v(g)| \\
& =\sup _{\substack{|g| \leq f^{2} \\
g \in \mathscr{K}_{C}(S)}}|v(g)| .
\end{aligned}
$$

On the other hand

$$
\begin{aligned}
\mu \cdot(f)= & \sup _{\substack{|g| \leq t \\
g \in \mathscr{K}_{C}(S)}}\|\mu(g)\|=\sup _{\substack{|g| \leq f \\
g \in \mathscr{K}_{C}(S)}} B(g, \bar{g})^{1 / 2} \\
= & \sup _{\substack{|g| \leq t \\
g \in \mathscr{K}_{C}(S)}} v\left(|g|^{2}\right)^{1 / 2} .
\end{aligned}
$$

Since

$$
\sup _{\substack{|g| \leq t \\ g \in \mathscr{K}_{C}(S)}} v\left(|g|^{2}\right) \leq \sup _{\substack{|g| \leq f^{2} \\ g \in \mathscr{K}_{C}(S)}}|v(g)|
$$

we get

$$
\mu^{\bullet}(f) \leq|v| \cdot\left(f^{2}\right)^{1 / 2} .
$$

Moreover, since $v$ is positive we get 


$$
|v(h)|=|v(\operatorname{Re} h)+i v(\operatorname{Im} h)| \leq 2 v(|h|)
$$

for all $h \in \mathscr{K}_{C}(S)$. Thus

$$
\begin{aligned}
|v|^{\bullet}\left(f^{2}\right)^{1 / 2} & \leq\left(2 \sup _{\substack{|g| \leq f^{2} \\
g \in \mathscr{K}_{C}(S)}} v(|g|)\right)^{1 / 2}=2^{1 / 2} \sup _{\substack{|g| \leq f^{2} \\
g \in \mathscr{K}_{C}(S)}} v(|g|)^{1 / 2} \\
& =2^{1 / 2} \sup _{\substack{|h| \leq f \\
h \in \mathscr{K}_{C}(S)}} v\left(|h|^{2}\right)^{1 / 2}=2^{1 / 2} \mu^{\bullet}(f) .
\end{aligned}
$$

The relation (14) is thus proved for all $f \in \mathscr{J}_{+}(S)$.

A straightforward calculation shows that the relation (14) is satisfied for all $f: S \rightarrow R^{+} \cup\{\infty\}$ with compact support and therefore even for all $f: S \rightarrow R^{+} \cup\{\infty\}$.

The relation $\mathscr{L}_{C}^{1}(\mu)=\mathscr{L}_{C}^{2}(v)$ is a direct consequence of the relation (14) and of the definitions of the spaces $\mathscr{L}_{C}^{1}(\mu)$ and $\mathscr{L}_{C}^{2}(v)$.

To show that

$$
B(f, g)=\int f \bar{g} d v \quad \text { for all } f, g \in \mathscr{L}_{C}^{1}(\mu)
$$

it is enough to note that the assumptions stated in Theorem 17 are satisfied, by Lemma 20, for all $f, g \in \mathscr{L}_{C}^{1}(\mu)$.

The theorem is proved.

Acknowledgement. This work has been supported by the Magnus Ehrnrooth Foundation. The author also wants to thank Professor I.S. Louhivaara and Dr. Kari Ylinen for inspiring conversations.

\section{References}

[1] Bourbaki, N.: Éléments de mathématique. XIII. Intégration. Chapitres 1-4. - Actualités Sci. Indust. 1175, Hermann, Paris, 1952 / 1965.

[2] -》- Éléments de mathématique. XXI. Intégration. Chapitre 5. 2ième éd. - Actualités Sci. Indust. 1244, Hermann, Paris, 1967.

[3] EDWARDS, D. A.: Vector-valued measure and bounded variation in Hilbert space. - Math. Scand. 3, 1955, 90-96.

[4] Masani, P.: Orthogonally scattered measures. - Advances in Math. 2, 1968, $61-117$.

[5] Morse, M., and W. Transue: $C$-bimeasures $A$ and their integral extensions. Ann. of Math. (2) 64, 1956, 480-504.

[6] Musiat, K.: Orthogonally scattered measures of $\sigma$-finite variation. - To appear in Transactions of the Seventh Prague Conference of Information Theory, Statistical Decision Functions, Random Processes, 1974.

[7] NIEMI, H.: Stochastic processes as Fourier transforms of stochastic measures. Ann. Acad. Sci. Fenn. Ser. A I 591, 1975, 1-47. 
[8] Sundaresan, K., and W. A. Woyczynski: $L$-orthogonally scattered measures. Vector and operator valued measures and applications, edited by D. H. Tucker and H. B. Maynard, pp. 343-350. - Academic Press, Inc., New York and London, 1973.

[9] Thomas, E.: L'intégration par rapport à une mesure de Radon vectorielle. Ann. Inst. Fourier (Grenoble) 20:2, 1970, 55-191.

[10] Varopoulos, N. Th.: Tensor algebras and harmonic analysis. - Acta Math. $119,1967,51-112$.

\author{
University of Helsinki \\ Department of Mathematics \\ SF-00100 Helsinki 10 \\ Finland
}

Received 21 April 1975 